\title{
Carcinoma-like nonfunctional pheochromocytoma in the right adrenal gland: A case report
}

\author{
SHINGO MORIYAMA $^{1}$, HIDEKI TAKESHITA ${ }^{1}$, SAORI ARAKI $^{1}$, TAKUO TOKAIRIN $^{2}$, \\ MAKOTO KAGAWA $^{1}$, KOJI CHIBA ${ }^{1}$, AKIKO ADACHI $^{2}$ and AKIRA NORO ${ }^{1}$ \\ Departments of ${ }^{1}$ Urology and ${ }^{2}$ Pathology, Saitama Red Cross Hospital, Saitama 338-8553, Japan
}

Received March 24, 2015; Accepted April 12, 2016

DOI: $10.3892 / 01.2016 .4776$

\begin{abstract}
Evaluation of the malignant potential of a pheochromocytoma (PCC) remains controversial. PCC is regarded as a neuroendocrine tumor (NET), and the classification of NETs has gradually been defined over the last decade, particularly for gastroenteropancreatic NET. The present study describes a case of locally advanced, carcinoma-like, nonfunctional PCC, which may be regarded as neuroendocrine carcinoma (NEC) rather than a malignant PCC. A 72-year-old man was referred to Saitama Red Cross Hospital (Saitama, Japan), presenting with a 2-month history of right flank pain. Computed tomography revealed a right adrenal gland tumor, which measured $6.0 \mathrm{~cm}$ in diameter, invading the hilum of the right kidney, liver and inferior vena cava (IVC). Radical surgery was performed with en bloc resection of the right kidney, and adjacent parts of the liver and IVC. Immunohistochemical examination demonstrated that all of the resected tissues were positive for cytokeratin AE1/AE3, chromogranin A, synaptophysin, cluster of differentiation 56 and Ki-67, and the specimen had a Ki-67 index of $80 \%$. A diagnosis of carcinoma-like PCC or NEC of the adrenal gland was confirmed. Reports of NEC of the adrenal gland are extremely rare in the literature, and classification of PCC as a NET has not yet been fully discussed. The present case may therefore contribute to the classification of NETs in the adrenal gland.
\end{abstract}

\section{Introduction}

Pheochromocytoma (PCC) is regarded as a form of neuroendocrine tumor (NET) $(1,2)$. PCC and NETs are extremely similar in terms of tumor cell origin, clinical and pathological features, and malignant potential $(1,2)$. PCC arises from chromaffin cells

Correspondence to: Dr Shingo Moriyama, Department of Urology, Saitama Red Cross Hospital, 8-3-33 Kamiochiai, Chuo, Saitama 338-8553, Japan

E-mail: zodiac_507@hotmail.com

Key words: neuroendocrine tumor, neuroendocrine carcinoma, adrenal gland neoplasms, Ki-67, pheochromocytoma, malignant pheochromocytoma in the adrenal medulla and typically produces catecholamines, resulting in characteristic symptoms, including hypertension, hyperglycemia and headaches (1). PCC without symptoms or secretion of catecholamines, which has been generally termed nonfunctional PCC, is extremely rare (3).

PCC has malignant potential similar to other NETs (4). Generally, $10 \%$ of all PCC cases develop metastases in other areas of the body such as lymph node, bone, liver and lung, and are commonly diagnosed as malignant at the time that metastases appear $(1,4)$. It is difficult to diagnose malignant PCC prior to the appearance of metastases due to the similarity of pathological features between benign and malignant PCC $(5,6)$. Thus, the diagnosis of malignancy and classification of PCC have remained under discussion.

The present case described a 72-year-old man with locally advanced, carcinoma-like, nonfunctional PCC of the adrenal gland, which appeared to be more similar to a neuroendocrine carcinoma (NEC) than a malignant PCC. The current study also discussed the malignant potential of PCC and NETs, and examined the potential application of the gastroenteropancreatic NET (GEP-NET) classification to the adrenal gland in evaluation of malignancy.

\section{Case report}

A 72-year-old man, who was followed up for non-muscle-invasive bladder cancer [urothelial carcinoma, grade 3>2, pT1, according to World Health Organization (WHO) 1973 classification system (7)] with no recurrence or metastasis for 2 years, was referred to Saitama Red Cross Hospital (Saitama, Japan) in August 2012, presenting with a 2-month history of right flank pain. A computed tomography scan (Aquilion; Toshiba Medical Systems Co., Ltd., Otawara, Japan) revealed a right adrenal tumor, measuring $6.0 \mathrm{~cm}$ in diameter, with an irregular and partially nodular outline. The tumor extended to the inferior vena cava (IVC), hilum of the right kidney and liver (Fig. 1). Iodine-123 metaiodobenzylguanidine (MIBG) scintigraphy results were negative, and all serum adrenal hormone levels were within normal limits; however, a slightly elevated level of the tumor marker neuron-specific $\gamma$-enolase $(25.7 \mathrm{ng} / \mathrm{ml}$; normal range, $<10 \mathrm{ng} / \mathrm{ml}$ ) was observed. These imaging and laboratory findings suggested the presence of adrenal gland cancer without metastasis. Radical surgery was performed with en bloc resection of the tumor by removing the right kidney, 
A

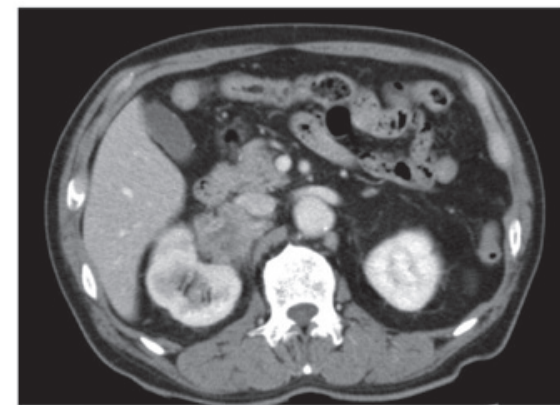

B

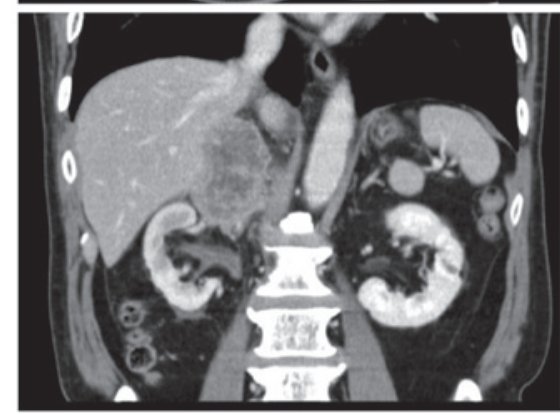

Figure 1. Abdominal contrast-enhanced computed tomography showing a right adrenal tumor $(6.0 \mathrm{~cm}$ in diameter) (A) suspected of invading the adjacent liver, and (B) extending into the posterior of the inferior vena cava and the hilum of the right kidney.

and adjacent areas of the liver and IVC. The specimen was fixed with $20 \%$ formalin and embedded in paraffin. Sections (3- $\mu \mathrm{m}$ thick) were cut and stained with hematoxylin and eosin. Immunohistochemistry was performed using the avidin-biotinperoxidase-complex method with antibodies against cytokeratin (CK) AE1/AE3 (mouse monoclonal antibody, diluted 1:1; Nichirei Biosciences Inc., Tokyo, Japan), chromogranin A (rabbit polyclonal antibody, diluted 1:1; Nichirei Biosciences Inc.), synaptophysin (mouse monoclonal antibody, diluted 1:1; catalogue number 27G12; Nichirei Biosciences Inc.), cluster of differentiation (CD) 56 (mouse monoclonal antibody, diluted 1:1; catalogue number 1B6; Nichirei Biosciences Inc.) and Ki-67 (rabbit polyclonal antibody, diluted 1:1; catalogue number SP6; Nichirei Biosciences Inc.). Pathological examination revealed that the tumor cells were arranged in cord-like and alveolar patterns, and exhibited a high nucleus-to-cytoplasm ratio with abundant nuclear chromatin. Immunohistochemical staining was positive for CKAE1/AE3, chromogranin A, synaptophysin, CD56 and Ki-67. The mitotic count was 27/10 high-power fields (HPFs), and the Ki-67 index was $80 \%$ (Fig. 2). The pathological diagnosis was confirmed as carcinoma-like PCC or NEC of the right adrenal gland. The tumor also invaded the IVC and metastasized to the lymph nodes surrounding the IVC. Adjuvant chemotherapy for this type of tumor is not known to be effective (8); therefore, the patient was observed postoperatively and experienced no recurrence after 9 months with follow-ups every 3 months. Informed consent was obtained from the patient for publication of the present case report.

\section{Discussion}

NETs may appear in a number of locations in the body, and are commonly observed in the lungs, pancreas and gastrointestinal tract (9). NETs originate from neuroendocrine

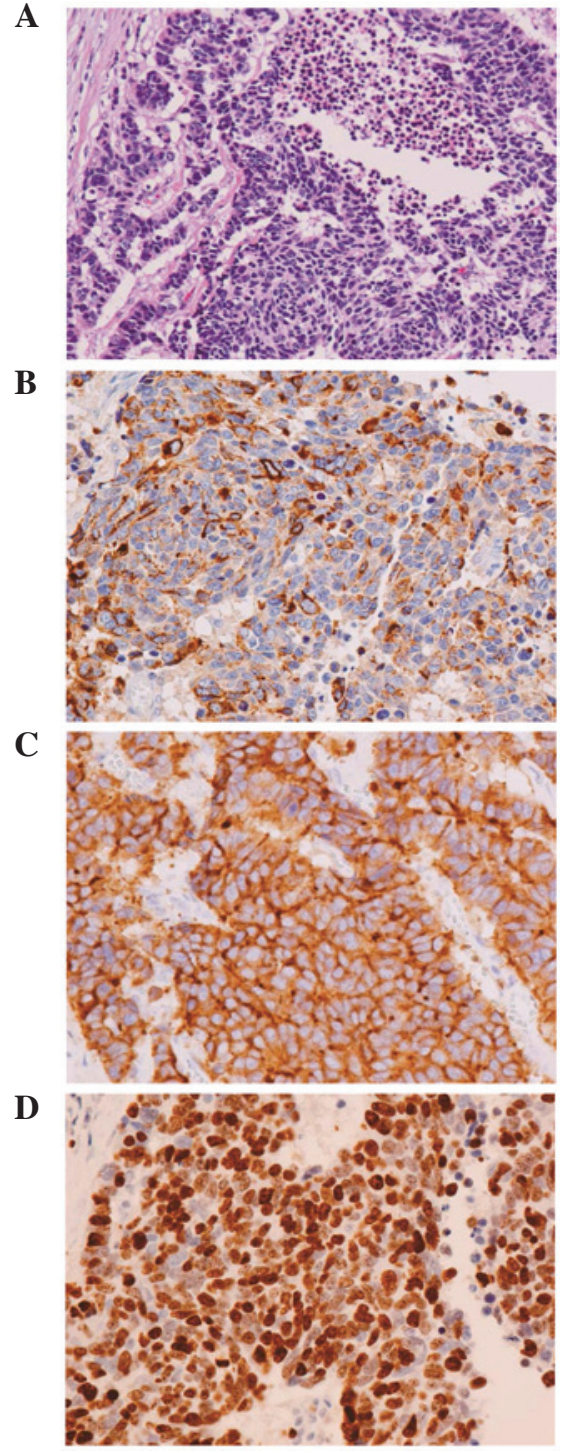

Figure 2. A histopathological diagnosis of neuroendocrine carcinoma of the adrenal gland was confirmed based on (A) H\&E staining (magnification, $x 20$ ), and immunohistochemical staining positive for (B) chromogranin A (magnification, x40), (C) synaptophysin (magnification, x40) and (D) Ki-67 (magnification, $\mathrm{x} 40$ ). H\&E staining revealed that the polygonal tumor cells had a high nucleus-to-cytoplasm ratio with abundant nuclear chromatin, and were arranged in cord-like and alveolar patterns. The mitotic count was 27/10 high-power fields, while the Ki-67 index was $80 \%$. H\&E, hematoxylin and eosin.

cells, and the overproduction of hormones within the tumor results in characteristic symptoms, which are dependent on the unique effect of each hormone (2). NETs have been considered as malignant tumors as they are able to metastasize long after treatment (10). However, a number of different organ-specific diagnostic systems have been developed for each NET origin; therefore, the staging and grading of NETs remains unclear (11).

PCC also has specific grading systems for malignancy. The diagnosis of malignant PCC prior to the appearance of metastases is considered difficult due to the histological similarities with benign PCC $(5,6)$. There are certain grading systems, including Pheochromocytoma of the Adrenal Scaled Score (5) and Grading system for Adrenal Pheochromocytoma and Paraganglioma (6), used to characterize the malignancy of PCC $(4,5)$. 
Generally, scoring systems are very useful for risk assessment, particularly for predicting the probability of recurrence or metastasis. However, they are a little cumbersome as diagnostic tools to discriminate malignancy from benign tumor in a clinical setting, since they require 12 or 6 pathological factors for their individual scoring systems. Furthermore, these scoring systems fully depend on the pathological findings, and this is the cause of variance in judging each feature among pathologists (12). Therefore, they have not yet had a significant impact on changes in pathological diagnosis and follow-up management. Previous studies have suggested that the Ki-67 index may be valuable in the diagnosis of malignant PCC (13-15). The classification using the $\mathrm{Ki}-67$ index alone may be considered as simple and easy to apply in the clinical setting. However, the majority of malignant PCCs exhibit lower Ki-67 index than general malignant tumors, and setting a clear cut-off value within a narrow range $(<5 \%)$ appears to be difficult (16). The cut-off value of Ki-67 index remains controversial. Therefore, a Ki-67 index-based classification of PCC would require careful consideration prior to application.

$\mathrm{NEC}$ is a rare form of NET with a high grade of malignancy (17). However, the definition of NEC remains unclear due to its rarity and diversity (17). NECs include a number of different tumors that originate in various organs and occasionally co-exist with another malignancy such as adenocarcinoma or urothelial carcinoma in the same specimen, which makes it difficult to clearly categorize (18). However, to the best of our knowledge, the majority of NEC cases are small cell carcinoma (SCC), and $\sim 95 \%$ of all SCCs originate from the lungs (19). Therefore, extrapulmonary NECs are extremely rare.

In 2010, the WHO advocated a simple and high-impact classification of GEP-NETs (17). The classification system categorized GEP-NET into three groups according to mitotic count and Ki-67 index as follows: ii) NET grade 1 (low-grade malignancy); ii) NET grade 2 (intermediate-grade malignancy); and iii) NEC (high-grade malignancy, described as carcinoma) (17). NEC is defined by a mitotic count of $>20 / 10$ HPFs or a Ki-67 index of $>20 \%$ (17). This classification system is simple and has been proven useful for predicting survival; therefore, it has been gradually accepted in pathology, digestive internal medicine and surgery (20). NETs in various organs share a number of common pathological features; therefore, whether the GEP-NET classification can be applied to other organs is now under careful consideration (12).

The present case was considered to be more similar to NEC of the adrenal gland than malignant PCC. Although the tumor localization and the immunohistochemical staining indicated that this tumor had arisen from the adrenal medulla, the tumor lacked several clinical features of PCC, such as an accumulation on iodine-123 MIBG scintigraphy and overproduction of catecholamines. Scintigraphy using iodine-123 MIBG, which mimics noradrenaline, is generally able to detect PCC (1), but the current case demonstrated negative results. Nonfunctional PCC, which refers to non-secreting PCC in the present study, is known to exist, but is extremely rare (3). Nonfunctional PCC, which may be associated with succinate dehydrogenase subunit B mutations (3), has not yet been clearly defined. Certain authors understand that nonfunctional PCC means "PCC without any symptoms", regardless of the overexpression of catecholamine, and it may be occasionally described as asymptomatic PCC or unsuspected PCC. Furthermore, a number of oncocytic PCCs may be regarded as nonfunctional PCC, and nonfunctional adenomas that are not resected or biopsied may contain nonfunctional PCCs. Therefore, it is not possible to clearly state the definite number of cases of "nonfunctional (non-secreting) PCC", and only a few studies have reported cases of nonfunctional or non-secreting PCC (21-23). Altogether, nonfunctional PCC itself is extremely rare, and malignant change or anomaly of nonfunctional PCC is no longer inconceivable; therefore, the present case may not be classified as malignant PCC.

NEC may also be diagnosed following identification of distinctive immunohistochemical staining, including chromogranin A, synaptophysin and CD56. NEC is reportedly strongly positive for CKs such as CK18, AE1/AE3 and CAM 5.2, while PCC is typically negative $(24,25)$. In the present case, all CK and neuroendocrine markers were positive. This pathological finding provided support for the diagnosis of NEC, and the tumor in the present case appears to be different from the malignant PCC generally observed among $10 \%$ of all PCCs. Furthermore, the present case of carcinoma-like PCC may be regarded as a type of SCC of the adrenal gland. However, SCC may account for a large number of NEC cases. Therefore, the tumor in the present case was preferably described as NEC of the adrenal gland, since PCC is a form of NET.

To the best of our knowledge, only 2 cases of NEC of the adrenal gland have been reported $(26,27)$. If the GEP-NET classification system is applied to adrenal medulla tumors, then PCC would be equivalent to NET grade 1, malignant PCC would be equivalent to NET grade 2 and carcinoma-like tumors (as in the current case) would be equivalent to NEC. The versatility of this NET classification requires further examination and clarification in future investigations.

In conclusion, the current study presented an extremely rare case of carcinoma-like, nonfunctional PCC, which may have been considered as more similar to NEC of the adrenal gland. To the best of our knowledge, there are currently no reports clearly indicating that NECs are the same as carcinoma-like PCC. However, small cell carcinoma may account for a large number of NEC cases, and carcinoma-like PCC may be a form of small cell carcinoma of the adrenal gland; this appears different from the malignant PCC generally observed among $10 \%$ of all PCCs. Therefore, the tumor in the present case was diagnosed as a carcinoma-like PCC, but was preferably described as NEC of the adrenal gland. Such cases are extremely rare, and conclusive evidence was not available in the current case; however, it is considered that these findings will contribute to further investigation of malignancies in the adrenal gland.

\section{References}

1. Strosberg JR: Update on the management of unusual neuroendocrine tumors: Pheochromocytoma and paraganglioma, medullary thyroid cancer and adrenocortical carcinoma. Semin Oncol 40: 120-133, 2013.

2. Kulke MH, Shah MH, Benson AB III, Bergsland E, Berlin JD, Blaszkowsky LS, Emerson L, Engstrom PF, Engstrom PF, Fanta P, et al; National comprehensive cancer network: Neuroendocrine tumors, version 1.2015. J Natl Compr Canc Netw 13: 78-108, 2015

3. Mannelli M, Lenders JW, Pacak K, Parenti G and Eisenhofer G: Subclinical phaeochromocytoma. Best Pract Res Clin Endocrinol Metab 26: 507-515, 2012. 
4. Korevaar TI and Grossman AB: Pheochromocytomas and paragangliomas: Assessment of malignant potential. Endocrine 40: 354-365, 2011.

5. Modlin IM, Oberg K, Chung DC, Jensen RT, de Herder WW, Thakker RV, Caplin M, Delle Fave G, Kaltsas GA, Krenning EP, et al: Gastroenteropancreatic neuroendocrine tumours. Lancet Oncol 9: 61-72, 2008.

6. Thompson LD: Pheochromocytoma of the Adrenal gland Scaled Score (PASS) to separate benign from malignant neoplasms: A clinicopathologic and immunophenotypic study of 100 cases Am J Surg Pathol 26: 551-566, 2002.

7. Mostofi FK, Sobin LH and Torloni H: Histological typing of urinary bladder tumours. In: International Histological Classification of Tumours. Vol 10. World Health Organization, Geneva, 1973.

8. Strosberg JR, Coppola D, Klimstra DS, Phan AT, Kulke MH, Wiseman GA and Kvols LK; North American Neuroendocrine Tumor Society (NANETS): The NANETS consensus guidelines for the diagnosis and management of poorly differentiated (highgrade) extrapulmonary neuroendocrine carcinomas. Pancreas 39 : 799-800, 2010

9. Kimura N, Watanabe T, Noshiro T, Shizawa S and Miura Y: Histological grading of adrenal and extra-adrenal pheochromocytomas and relationship to prognosis: A clinicopathological analysis of 116 adrenal pheochromocytomas and 30 extra-adrenal sympathetic paragangliomas including 38 malignant tumors. Endocr Pathol 16: 23-32, 2005.

10. Massironi S,Sciola V,Peracchi M,Ciafardini C,SpampattiMP and Conte D: Neuroendocrine tumors of the gastro-entero-pancreatic system. World J Gastroenterol 14: 5377-5384, 2008.

11. Klimstra DS, Modlin IR, Coppola D, Lloyd RV and Suster S: The pathologic classification of neuroendocrine tumors: A review of nomenclature, grading, and staging systems. Pancreas 39 707-712, 2010

12. Wu D, Tischler AS, Lloyd RV, DeLellis RA, de Krijger R, van Nederveen $F$ and Nosé V: Observer variation in the application of the Pheochromocytoma of the Adrenal Gland Scaled Score. Am J Surg Pathol 33: 599-608, 2009.

13. Clarke MR, Weyant RJ, Watson CG and Carty SE: Prognostic markers in pheochromocytoma. Hum Pathol 29: 522-526, 1998.

14. van der Harst E, Bruining HA, Jaap Bonjer H, van der Ham F, Dinjens WN, Lamberts SW, de Herder WW, Koper JW, Stijnen T, Proye C, et al: Proliferative index in phaeochromocytomas: Does it predict the occurrence of metastases? J Pathol 191: 175-180, 2000.

15. Boltze C, Mundschenk J, Unger N, Schneider-Stock R, Peters B, Mawrin C, Hoang-Vu C, Roessner A and Lehnert H: Expression profile of the telomeric complex discriminates between benign and malignant pheochromocy toma. J Clin Endocrinol Metab 88: 4280-4286, 2003.
16. Watanabe M: Malignant pheochromocytoma - Difficulties for diagnosis of malignancy. Official Journal of the Japan Association of Endocrine Surgeons and the Japanese Society of Thyroid Surgery 30: 41-44, 2013 (In Japanese).

17. Chang K, Dai B, Kong YY, Qu YY, Gan HL, Gu WJ, Ye DW, Zhang HL, Zhu Y and Shi GH: Genitourinary small-cell carcinoma: 11-year treatment experience. Asian J Androl 16: 705-709, 2014

18. Richardson RL and Weiland LH: Undifferentiated small cell carcinomas in extrapulmonary sites. Semin Oncol 9: 484-496, 1982.

19. Bosman FT, Carneiro F, Hruban RH and Theise ND: WHO Classification of Tumours of the Digestive System. IARC Press, Lyon, 2010.

20. Jann H,Roll S,Couvelard A,Hentic O,Pavel M,Müller-Nordhorn J, Koch M, Röcken C, Rindi G, Ruszniewski P, et al: Neuroendocrine tumors of midgut and hindgut origin: Tumor-node-metastasis classification determines clinical outcome. Cancer 117: 3332-3341, 2011

21. Gong J, Wang X, Chen X, Chen N, Huang R, Lu C, Chen D, Zeng $\mathrm{H}$ and Zhou Q: Adrenal and extra-adrenal nonfunctioning composite pheochromocytoma/paraganglioma with immunohistochemical ectopic hormone expression: Comparison of two cases. Urol Int 85: 368-372, 2010

22. Maurea S, Lastoria S, Cuocolo A, Celentano L and Salvatore M: The diagnosis of nonfunctioning pheochromocytoma. The role of I-123 MIBG imaging. Clin Nucl Med 20: 22-24, 1995.

23. Mannelli M, Pupilli C, Lanzillotti R, Ianni L, Amorosi A, Credi G and Pratesi C: A nonsecreting pheochromocytoma presenting as an incidental adrenal mass. Report on a case. J Endocrinol Invest 16: 817-822, 1993.

24. Gould VE, Wiedenmann B, Lee I, Schwechheimer K, Dockhorn-Dworniczak B, Radosevich JA, Moll R and Franke WW: Synaptophysin expression in neuroendocrine neoplasms as determined by immunocytochemistry. Am J Pathol 126: 243-257, 1987.

25. Chetty R, Pillay P and Jaichand V: Cytokeratin expression in adrenal phaeochromocytomas and extra-adrenal paragangliomas. J Clin Pathol 51: 477-478, 1998.

26. Ochiai T, Komiyama S, Ikoma H, Kubota T, Nakanishi M, Ichikawa D, Kikuchi S, Fujiwara H, Sakakura C, Kokuba Y, et al: A case report of metastatic neuroendocrine carcinoma of the right adrenal gland successfully treated with chemotherapy and surgery. Int J Clin Oncol 15: 423-427, 2010.

27. Juarez D, Brown RW, Ostrowski M, Reardon MJ, Lechago J and Truong LD: Pheochromocytoma associated with neuroendocrine carcinoma. A new type of composite pheochromocytoma. Arch Pathol Lab Med 123: 1274-1279, 1999. 